\title{
High-Pressure EPR Study of Spin Molecular Bistability in $\mathrm{Li}\left[\mathrm{Fe}(\mathrm{Th}-5 \mathrm{Cl}-\mathrm{Sa})_{2}\right]$
}

\begin{abstract}
A. KRUPSKA
Institute of Molecular Physics, Polish Academy of Sciences, M. Smoluchowskiego 17, 60-179 Poznań, Poland

(Received June 7, 2013; in final form April 22, 2014)

The spin transitions in $\mathrm{Li}\left[\mathrm{Fe}(\mathrm{Th}-5 \mathrm{Cl}-\mathrm{Sa})_{2}\right]$ were studied by EPR under hydrostatic pressure in the temperature range $80-332 \mathrm{~K}$. The structural changes in the complex anion induced by the pressure above $350 \mathrm{MPa}$ (when LS- 2 phase appears) are observed, which are manifested in abrupt changes in $g_{\perp}$ factor and linewidth $\Delta B_{\perp}$ of the low-spin phase LS. It is also manifested in the change in the shape of EPR spectrum. The high-spin $\leftrightarrow$ low-spin transition HS $\leftrightarrow$ LS shows hysteresis whose width is constant up to about $265 \mathrm{~K}$ and then rapidly increases. The spin phase diagram studied shows a large similarity to the phase diagram of $(2 \mathrm{Me}-5 \mathrm{Et}-\mathrm{PyH})\left[\mathrm{Fe}(\mathrm{Th}-5 \mathrm{Cl}-\mathrm{Sa})_{2}\right]$, which means that characteristics of the transition depend mainly on complex anion, not on cation. The hysteresis indicates on a cooperative character of the spin transition and on grouping of the complexes in domains.
\end{abstract}

DOI: 10.12693/APhysPolA.126.790

PACS: $75.30 . \mathrm{Wx}, 33.35 .+\mathrm{r}, 75.50 . \mathrm{Bb}$

\section{Introduction}

The comprehensive studies of a spin transition induced by changes of temperature, pressure, or light have been recently performed in iron(II) solid complexes [1-4]. Some of these compounds exhibit molecular bistability, which is attractive for eventual application in switching devices [4] or use as switches or sensors materials and for data recording [5]. Spin crossover of iron(III) complexes has been studied by magnetic susceptibility [6-9], heat capacity [6], the Mössbauer spectroscopy [6], or EPR spectroscopy [8-12].

Photoinduced spin transitions of iron(III) were investigated by magnetic susceptibility and the Mössbauer spectroscopy [13]. The iron(III) thiosemicarbazone spin-crossover and spin-transition properties were investigated by magnetic susceptibility, the Mössbauer spectroscopy, and differential scanning calorimetry measurements. Three compounds exhibit an abrupt spin transition with a thermal hysteresis effect [14]. The switching properties of the spin transition solid of iron(III) compounds have been investigated by using temperature and light $[15,16]$. Some molecular switching processes occurring in several types of inorganic solids due to temperature, light, pressure, and magnetic field have been described [17]. Photoinduced spin transition of $\mathrm{Fe}(\mathrm{II})$-triazole complex has been studied by the Mössbauer spectroscopy, UV and Fourier transform infrared spectroscopy [18]. Our EPR high pressure study of the spin transition of $\mathrm{Fe}$ (III) spin-crossover complexes in 2-methyl-5-ethyl piridine 5-chloro-salicylalthiosemicarbazonato ferrate, (2Me-5Et-PyH)[Fe(Th-5Cl-Sa $\left.)_{2}\right]$, revealed such bistability $[11,12]$.

This molecular system can exist in two different electronic states: high-spin ${ }^{6} A_{1}$ (HS) with $g_{\perp} \approx 2.21$ or

corresponding author; e-mail: krupska@ifmpan.poznan.pl low-spin ${ }^{2} T_{2}$ (LS) with $g_{\perp} \approx 2.16$ under pressure between 370 and $470 \mathrm{MPa}$ at room temperature. This complex behaviour under pressure can be expected for other isomorphic iron(III) compounds of thiosemicarbazonates of aromatic aldehydes with the general formula: $\mathrm{M}^{+}\left[\mathrm{Fe}(\mathrm{Th}-\mathrm{R}-\mathrm{Sa})_{2}\right]^{-}[6]$, where $\mathrm{M}=\mathrm{H}, \mathrm{Na}$, Cs or $2 \mathrm{Me}-5$ Et-PyH and $\mathrm{R}=\mathrm{Cl}$, Br. Previously our EPR studies concern the low spin domain formation in the process of spin transition in $\mathrm{Na}\left[\mathrm{Fe}(\mathrm{Th}-\mathrm{Sa})_{2}\right][8,9]$.

The studies of magnetic susceptibility, Mössbauer spectroscopy and heat capacity for the thiosemicarbazonates with the same complex anion [Fe(Th-5Cl-Sa $\left.)_{2}\right]$ and different cations revealed that their HS $\leftrightarrow$ LS transition temperature between 215 and $230 \mathrm{~K}$ does not differ so much [6]. It follows that the physical properties of the thiosemicarbazonates is determined mainly by structure of the complex anion. The confirmation of this conclusion should be obtained by the study of influence of the pressure on spin transition and comparison phase diagrams of the compounds with different cations.

In this paper we present the results of EPR studies of spin transitions in polycrystalline $\mathrm{Li}\left[\mathrm{Fe}(\mathrm{Th}-5 \mathrm{Cl}-\mathrm{Sa})_{2}\right]$ under high pressure in the wide temperature range. The main aim of this study is:

1. to determine the spin transition diagram and its relation with the structural phase transition induced by pressure;

2. to answer the question how the dimensions of the cation ion $\left(\mathrm{Li}^{+}\right.$instead of the complex $2 \mathrm{Me}-5 \mathrm{Et}-\mathrm{PyH}^{+}$) influences on the transition diagram as well as on temperature and pressure interval of the spin bistability.

\section{The structure of the Fe(III) complex}

The crystal structure of $\mathrm{Li}\left[\mathrm{Fe}(\mathrm{Th}-5 \mathrm{Cl}-\mathrm{Sa})_{2}\right]$ is not resolved. But, the structure of the complex anion is well known for isomorphic $\mathrm{NH}_{4}\left[\mathrm{Fe}(\mathrm{Th}-5 \mathrm{Cl}-\mathrm{Sa})_{2}\right]$, which crystallises in the rhombic Pnca space group with $Z=4$ [6]. 
We assume that the structure of $\mathrm{Fe}^{3+}$ complex for the whole family of $\mathrm{M}\left[\mathrm{Fe}(\mathrm{Th}-5 \mathrm{Cl}-\mathrm{Sa})_{2}\right]$ compounds is similar. The $\mathrm{Fe}^{3+}$ ion is always coordinated by two 5-chloro salicylaltiosemicarbazonato ONS-ligands in two mutually perpendicular planes with $\mathrm{O}$ and $\mathrm{S}$ atoms in cis and $\mathrm{N}$ atoms in trans positions. The molecular structure of the $\mathrm{Fe}^{3+}$ complex coordinated by two R-salicylaltiosemicarbazone ONS-ligands is presented in our earlier papers $[9,12]$. The $\mathrm{Fe}\left[\mathrm{O}_{2} \mathrm{~N}_{2} \mathrm{~S}_{2}\right]$ octahedron is in HS state bigger than in LS one and strongly distorted. The significant changes in the HS $\leftrightarrow$ LS transition are visible in the $\mathrm{Fe}^{3+}$-ligand distances as well as in the $\mathrm{L}-\mathrm{Fe}-\mathrm{L}$ angles.

\section{Experimental and results}

The powder sample of $\mathrm{Li}\left[\mathrm{Fe}(\mathrm{Th}-5 \mathrm{Cl}-\mathrm{Sa})_{2}\right]$ was prepared in the Moscow Physical Technical Institute according to procedure as described in [6]. The pressure EPR studies were performed applying special equipment previously described [19]. The measurements were carried out at different constant temperatures changing the pressure up and down between the atmospheric pressure and $550 \mathrm{MPa}$. The temperature measurements under atmospheric pressure were performed, too.

At room temperature under atmospheric pressure the powder EPR spectrum consists of a broad high spin HS signal at $g \simeq 2.1$, which is additionally deformed by paramagnetic impurities in the corundum resonator, used in measurements under pressure [19]. The resulting signal forms a background that can be approximated by two or three Lorentzian lines. Such as our previous paper $[11,12]$ this approximation does not have any physical sense; it only allows a separation of the background signal from the low spin LS spectrum by the numerical procedure. Because the intensity of the spurious signal from the resonator is less than $10 \%$ of the total intensity, thus that signal can be omitted in analysis of the spectrum. Here the background signal is treated as an intensity standard used to calculation of the LS intensity.

When the LS state of iron(III) complexes is observed, the summary spectrum can be approximated by:

1. LS state polycrystalline spectrum, obtained assuming the axial symmetry of $g$-factor and line-width, Lorentzian shape of the individual EPR line and taking into account only Zeeman term in a spin Hamiltonian, and

2. the background signal represented by three Lorentzian lines.

The numerical fitting procedure is applied, from which the LS state values of $g_{\perp}, g_{\|}$, slope linewidth $\left(\Delta B_{\perp}\right.$ and $\left.\Delta B_{\|}\right)$and intensity as well as the background data are obtained. Figure 1 shows the numerical analysis of the EPR spectrum for three selected \{temperature, pressure $\}$ points: $\{234 \mathrm{~K}, 60 \mathrm{MPa}\},\{233 \mathrm{~K}, 450 \mathrm{MPa}\}$ and $\{294 \mathrm{~K}, 460 \mathrm{MPa}\}$, which correspond to the coexistence region of two phases: HS $\leftrightarrow$ LS-1, LS-1 $\leftrightarrow$ LS-2, and $\mathrm{HS} \leftrightarrow \mathrm{LS}-2$, respectively. HS correspond to the high spin phase, LS-1 denotes the low pressure low spin phase and

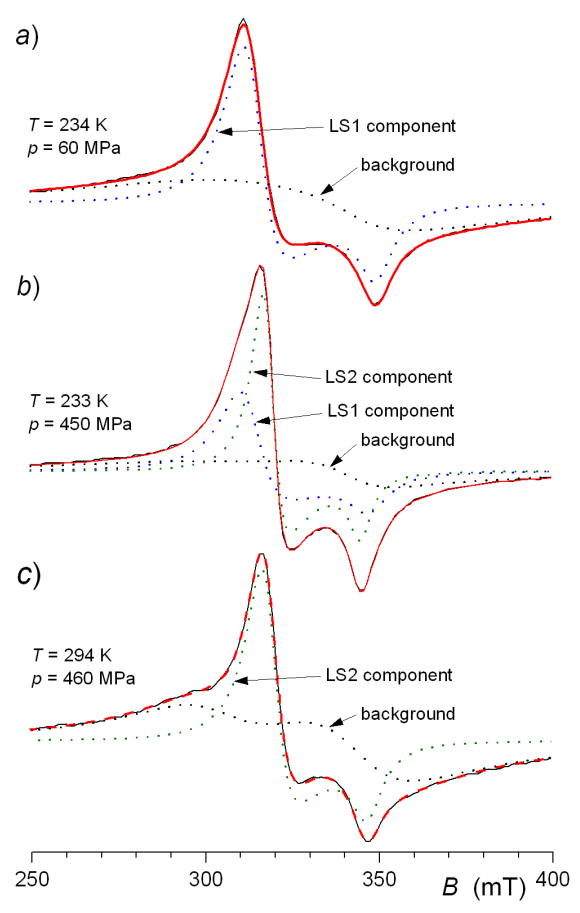

Fig. 1. Three examples of analysis of the EPR spectrum recorded: (a) at $234 \mathrm{~K}$ under pressure $60 \mathrm{MPa}$, (b) at $233 \mathrm{~K}$ under $450 \mathrm{MPa}$ and (c) $294 \mathrm{~K}$ under $460 \mathrm{MPa}$. The experimental spectra are given by solid black line. The solid red line represents the best fitted data to the experimental spectrum. The dotted lines are the components of the spectrum obtained from numerical fitting procedure.

LS-2 - high pressure low spin phase. The components of the spectrum: LS-1, LS-2 and background lines are plotted, too. Such fitting procedure was carried out for other recorded spectra.

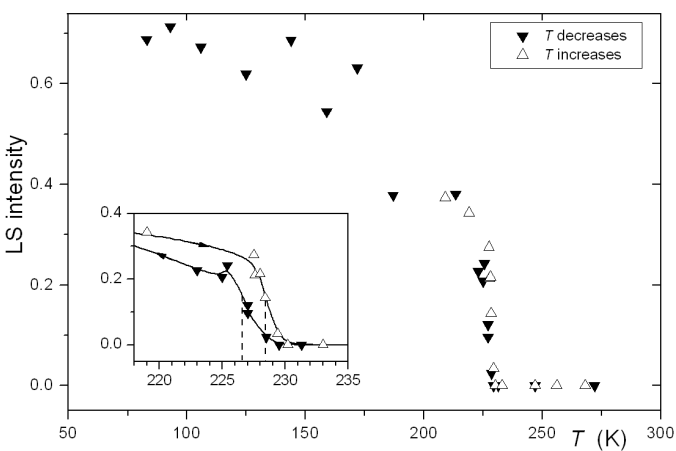

Fig. 2. The LS fraction for $\mathrm{Li}\left[\mathrm{Fe}(\mathrm{Th}-4 \mathrm{Cl}-\mathrm{Sa})_{2}\right]$ in dependence on temperature under atmospheric pressure. The inset shows changes of the LS intensity in the transition region and hysteresis loop. The solid lines indicate the phase transition temperatures $T_{\mathrm{c}}$ during decreases and increases of $T$.

Figure 2 presents the low-spin fraction as a function of temperature under atmospheric pressure. The low-spin fraction $\gamma_{\mathrm{LS}}$ is described by a formula: 


$$
\gamma_{\mathrm{LS}}=\frac{I_{\mathrm{LS}}}{I_{\mathrm{LS}}+I_{\mathrm{HS}}}
$$

where $I_{\mathrm{LS}}$ - LS intensity determined from the fitting procedure of the powder spectrum, and $I_{\mathrm{HS}}-$ high-spin intensity approximated by background signal intensity. This approximation is not quite true, because the treatment of the powder HS signal as a superposition of some Lorentzian lines is very rough. Nevertheless, we are convinced that this procedure gives correctly qualitative course of the intensity in dependence of temperature or pressure.

On cooling under atmospheric pressure the LS signal starts at $229 \mathrm{~K}$. The intensity of the signal initially rises abruptly and then the rise is gradually down to $83 \mathrm{~K}$. On warming the LS intensity decreases and abruptly change of the intensity occurs at $1.8 \mathrm{~K}$ higher than on cooling (see inset in Fig. 2). Reasonable is to define the transition temperature $T_{\mathrm{c}}$, in which half abrupt change of the intensity is observed, as it is seen in the inset by solid lines.
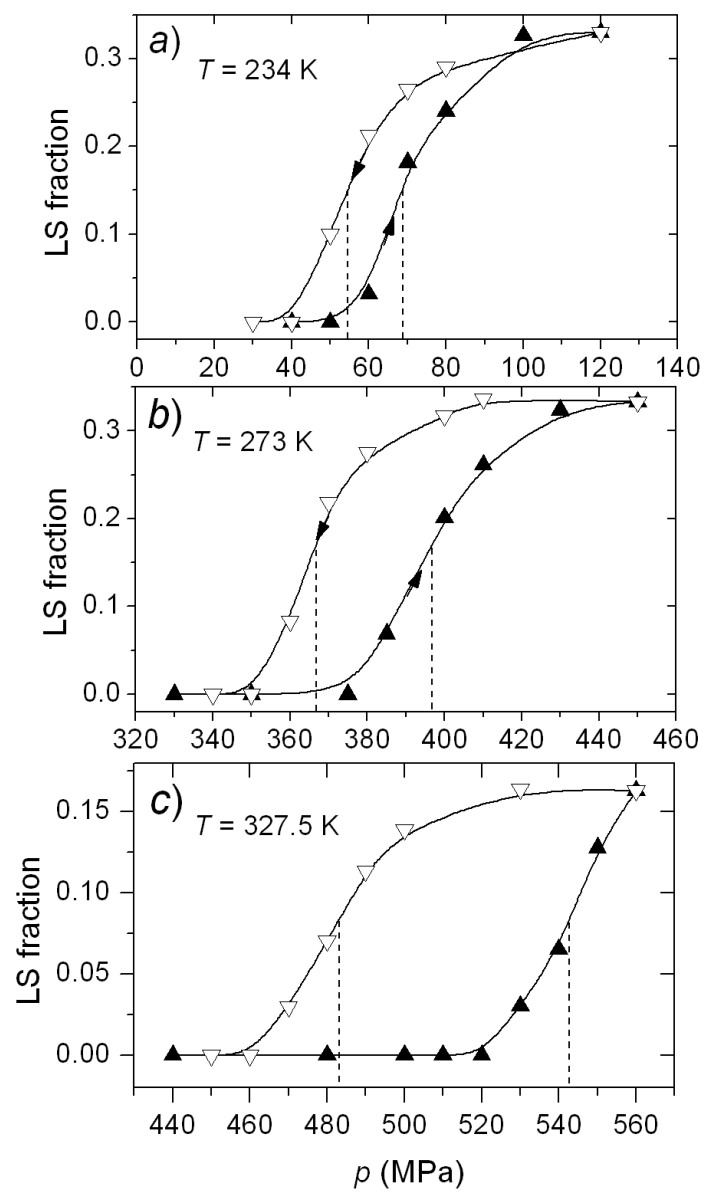

Fig. 3. The LS fraction of $\mathrm{Li}\left[\mathrm{Fe}(\mathrm{Th}-4 \mathrm{Cl}-\mathrm{Sa})_{2}\right]$ as a function of pressure determined at three temperatures in the region of the pressure hysteresis loops. The transition pressures $p_{\mathrm{c}}$ are denoted by dashed lines.

The further studies are carried out under hydrostatic pressure at several constant temperatures. Figure 3 shows the pressure dependence of the LS fraction for the HS $\leftrightarrow$ LS transition at three chosen temperatures: 234,273 , and $327.5 \mathrm{~K}$ during increases and decreases of pressure. The hysteresis loop is visible. The pressure measurements are restricted to the region of the pressure when abrupt changes of the LS intensity are observed. The pressure transition $p_{\mathrm{c}}$ is defined like as $T_{\mathrm{c}}$ and denoted by dashed lines.

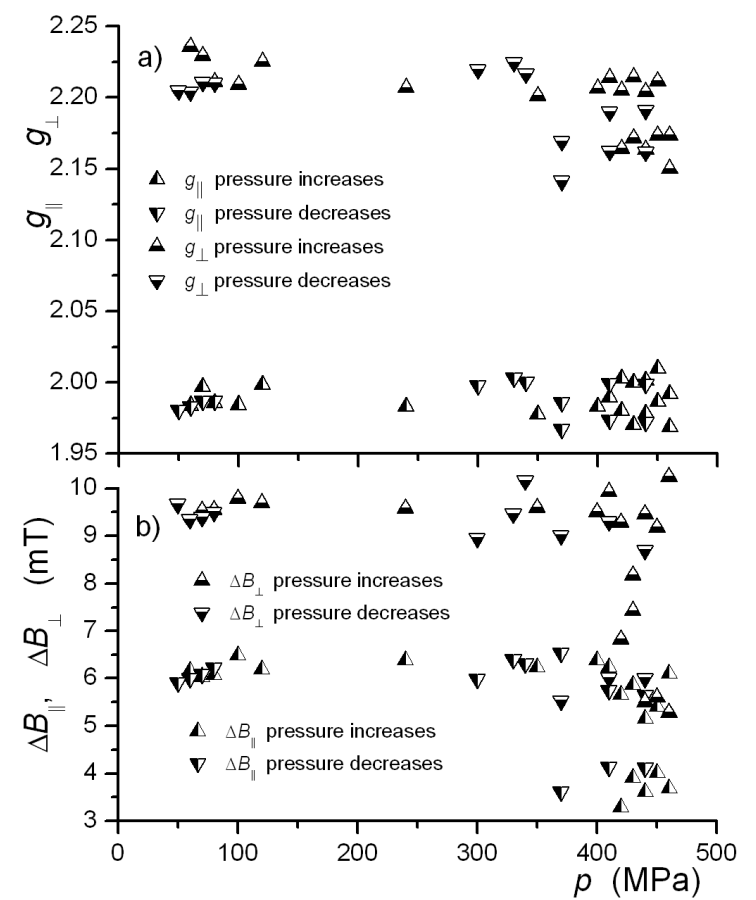

Fig. 4. The pressure dependence for $\mathrm{Li}[\mathrm{Fe}(\mathrm{Th}-4 \mathrm{Cl}-$ -Sa $\left.)_{2}\right]$ of (a) $g_{\perp}$ and $g_{\|}$components and (b) $\Delta B_{\perp}$ and $\Delta B_{\|}$obtained from the fitting procedure.

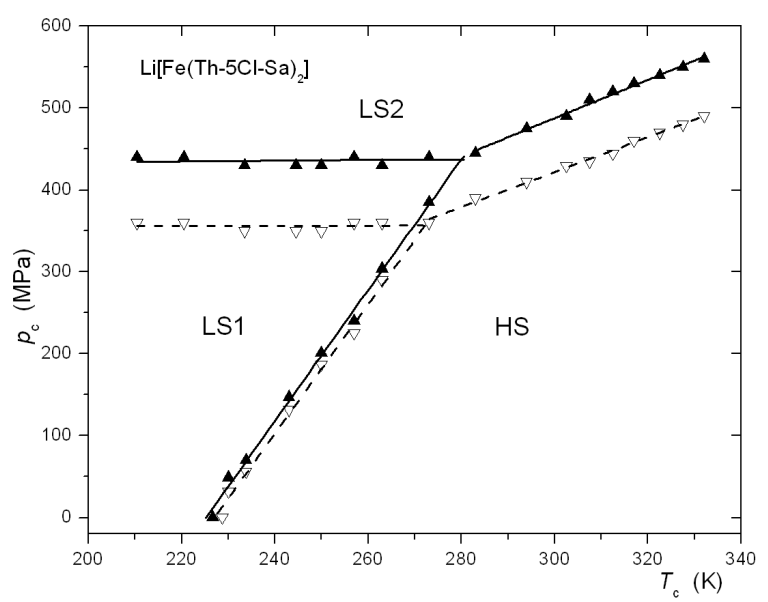

Fig. 5. The phase diagram $p(\mathrm{MPa})$ versus $T(\mathrm{~K})$ for $\mathrm{Li}\left[\mathrm{Fe}(\mathrm{Th}-5 \mathrm{Cl}-\mathrm{Sa})_{2}\right]$ compound.

As it is seen from Fig. 1b the LS-1 and LS-2 EPR spectra exhibit different $g$-values. The $g$-values and line width $\Delta B$ obtained from the fitting procedure are plotted as a function of pressure in Fig. 4. The mean values are: 
$g_{\|}=1.99, g_{\perp}=2.21, \Delta B_{\|}=6.25$, and $\Delta B_{\perp}=9.7$ for LS-1 phase and $g_{\|}=1.98, g_{\perp}=2.16, \Delta B_{\|}=3.75$ and $\Delta B_{\perp} \approx 6.0$ for LS-2 phase.

Figure 5 presents the phase diagram of pressure $p$ ( $\mathrm{MPa}$ ) versus temperature $T(\mathrm{~K})$ by EPR method defined. As follows from the diagram the LS-1 $\leftrightarrow$ LS-2 (low spin-1 phase to low spin-2 phase) transition shows the large pressure hysteresis of $80 \mathrm{MPa}$ up to $273 \mathrm{~K}$.
Above that temperature the value of the hysteresis is about $65 \mathrm{MPa}$.

As follows from Fig. 5 and Fig. 4 the rapid changes of the $g_{\perp}, \Delta B_{\perp}$, as well as in the intensity above $273 \mathrm{~K}$ are observed. These parameters distinctly decrease above $273 \mathrm{~K}$. The parameters obtained from the EPR study for $\mathrm{Li}\left[\mathrm{Fe}(\mathrm{Th}-5 \mathrm{Cl}-\mathrm{Sa})_{2}\right]$ at several temperatures are collected in Table I.

TABLE I

Parameters obtained from the EPR studies for $\mathrm{Li}\left[\mathrm{Fe}(\mathrm{Th}-5 \mathrm{Cl}-\mathrm{Sa})_{2}\right.$ for several temperatures. $P(\mathrm{LS}-2)$ up the pressure at which the high pressure low-spin phase appears, $P$ (LS-2) down - the pressure at which the high pressure low-spin phase disappears, $\Delta P 1 / 2$ his $(\mathrm{MPa})$ — width of hysteresis, $I$ (LS-2) max relatively maximum of relative intensity of EPR spectrum, $g_{\perp}$ - average value of spectroscopic splitting parameter $g$ at $z$ axis, $g_{\|}$- average value of spectroscopic splitting parameter $g$ at $x y$ axes, $\Delta B_{\perp}$ - line width at $z$ direction, $\Delta B_{\|}$- line width at $x y$ directions.

\begin{tabular}{c|c|c|c|c|c|c|c|c}
\hline \hline$T[\mathrm{~K}]$ & $\begin{array}{c}P(\mathrm{LS}-2) \\
\text { up } \\
\text { appear }\end{array}$ & $\begin{array}{c}P(\mathrm{LS}-2) \\
\text { down } \\
\text { disappear }\end{array}$ & $\begin{array}{c}\Delta P 1 / 2 \text { his } \\
{[\mathrm{MPa}]}\end{array}$ & $\begin{array}{c}I \text { (LS-2) } \\
\text { max. } \\
\text { relatively }\end{array}$ & $\begin{array}{c}g_{\perp} \\
\text { (average) }\end{array}$ & $\begin{array}{c}g_{\|} \\
\text {(average) }\end{array}$ & $\begin{array}{c}\Delta B_{\perp} \\
\text { (average) }\end{array}$ & $\begin{array}{c}\Delta B_{\|} \\
\text {(average) }\end{array}$ \\
\hline 230 & 35 & 25 & 8.48 & 10.5 & 2.215 & 1.995 & 9.53 & 5.86 \\
233 & 65 & 40 & 10.29 & 7.81 & 2.216 & 1.98 & 9.56 & 6.13 \\
243 & 140 & 125 & 15.45 & $7.5 / 6.9$ & 2.2 & 1.97 & 9.24 & 6.02 \\
250 & 190 & 180 & 11.93 & $7.33 / 7.07$ & 2.21 & 1.97 & 10.46 & 6.79 \\
263 & 300 & 280 & 17.39 & 7.89 & 2.22 & 1.98 & 10.87 & 6.93 \\
273 & 390 & 350 & 47.5 & 14.17 & 2.2 & 1.99 & 10.48 & 6.46 \\
283 & 440 & 385 & 53.57 & 4.05 & 2.16 & 1.95 & 6.79 & 4.63 \\
294 & 470 & 395 & 68.05 & 6.44 & 2.17 & 1.98 & 6.94 & 5.22 \\
\end{tabular}

\section{Discussion}

The Fe(II) and Fe(III) spin-crossover compounds reversibly switch between a diamagnetic LS state and a paramagnetic HS state under temperature, hydrostatic pressure, or light. The transitions between low-spin state and high spin state usually are very abrupt with a wide hysteresis loops. As follows from Fig. 3 the pressure hysteresis associated with a spin transition is observed. A similar hysteresis associated with a thermal hysteresis was observed in the iron(II) compounds [20]. The similar thermal hysteresis connected with abrupt spin transition has been recently observed for three neutral thiosemicarbazone ferric complexes of general formulae $[\mathrm{Fe}(\mathrm{H} 5 \mathrm{XThSa})(5 \mathrm{XThSa})] \cdot \mathrm{H}_{2} \mathrm{O}[21]$. The related effect has been also observed in other type of the thiosemicarbazones compounds. Abrupt spin transition with a thermal hysteresis has been observed for three 5X-salicylaldehyde thiosemicarbazone [14]. This type compounds where hysteresis occurs are the most attractive to a memory effect in magnetic devices, developing switches, or sensors [5].

We suppose that the hysteresis is connected with domain formation in the LS phases and their sizes. In our earlier studies, the EPR line-width analysis showed that LS complexes aggregate in domain, which is strictly connected with the presence of hysteresis [9]. Based on the theory of Gütlich for iron(II) compounds, thermodynamic analysis carried out recently for pressure-induced spin transitions in thiosemicarbazonates of iron(III) showed the close relationship between the size of domain and hysteresis [22].

The changes obtained in some parameters $\left(g_{\perp}, \Delta B_{\perp}\right)$ are probably connected with the structural changes in complexes in a perpendicular direction. The similar changes in a perpendicular direction that we have observed in our previous compound contains the piridine cation $[11,12]$.

The temperatures of the HS $\leftrightarrow$ LS transition for the isomorphic compounds $\mathrm{M}^{+}\left[\mathrm{Fe}(\mathrm{Th}-5 \mathrm{Cl}-\mathrm{Sa})_{2}\right]^{-}$possessed the same complex anion with various cations are collected in Table II.

The conclusion which we may draw from Table II is that the spin transition temperature is almost independent of cation on condition that complex anion is the same. That means that spin transition depends mainly on structure of the complex anion.

As follows from Fig. 5 we obtain a new pressure-induced low-spin phase LS-2 called as a high pressure low spin phase. A similar effect has been obtained in the case of piridine cation [11, 12]. In the EPR spectra of $\mathrm{Li}\left[\mathrm{Fe}(\mathrm{Th}-5 \mathrm{Cl}-\mathrm{Sa})_{2}\right]$ the LS-1 signal is visible from 225.5 K (at ambient pressure). As follow from Fig. 5 the 
TABLE II

The temperature of the HS $\leftrightarrow$ LS transition of $\mathrm{M} .^{+}\left[\mathrm{Fe}(\mathrm{Th}-5 \mathrm{Cl}-\mathrm{Sa})_{2}\right]^{-}$compounds for various cations $\mathrm{M}^{+}$under atmospheric pressure.

\begin{tabular}{|c|c|c|}
\hline M. & $T_{\mathrm{c}}[\mathrm{K}]$ & Method \\
\hline $\mathrm{H}$ & $222 \div 226$ & $\begin{array}{c}\text { heat capacity [6] } \\
\text { magnetic susceptibility [7] } \\
\text { EPR [7] } \\
\text { Mössbauer spectra [6] }\end{array}$ \\
\hline $\mathrm{Li}$ & $\begin{array}{c}\approx 267 \\
227 \div 229\end{array}$ & $\begin{array}{c}\text { magnetic susceptibility [6] } \\
\text { EPR [this paper] }\end{array}$ \\
\hline $\mathrm{Na}$ & 208 & $\begin{array}{c}\text { magnetic susceptibility }[6,8,9] \\
\text { Mössbauer spectra [6] } \\
\text { EPR }[8,9]\end{array}$ \\
\hline $\mathrm{NH}_{4}$ & 220 & $\begin{array}{c}\text { magnetic susceptibility [6] } \\
\text { Mössbauer spectra [6] } \\
\text { EPR [10] }\end{array}$ \\
\hline $\mathrm{Al}_{0.33}$ & 228 & heat capacity [6] \\
\hline 2Me-5Et-PyH & 220 & $\begin{array}{c}\text { Mössbauer spectra [6] } \\
\operatorname{EPR}[11,12]\end{array}$ \\
\hline
\end{tabular}

phase transition does not depend on temperature and arises at the same pressure below the triple point. The similar situation has been observed in the case of piridine cation $[11,12]$. This fact allows supposition that also in this case some barrier of the Gibbs free energy $G$ exists and should be overcome to change the specific volume on $\Delta V=\partial G_{1} / \partial P-\partial G_{2} / \partial P$. The specificity of the phase transition in question consists in that transition occurs not in the whole volume of the initial HS phase but in the nucleus of the nucleating centre of a first LS-1 phase as in the piridine cation $[11,12]$.

We can suppose that thermodynamically first order phase transition occurs in the pressure induced low-spin phases (Fig. 3, Fig. 4). But appropriate studies would be needed to confirm the thermodynamic nature of these spin transitions.

\section{Conclusions}

- We can draw the general conclusion that hydrostatic pressure can induce a new low-spin phase (LS-2) in that category of iron(III) compounds.

- Spin transition depends mainly on structure of the complex anion.

- Below the triple point the phase transition does not depend on temperature and arises at the same pressure.

- Spin transition occurs in the nucleating centers of LS-1 phase.

- The hysteresis indicates on grouping of the complexes in domains.

\section{Acknowledgments}

I would like to thank late Prof. V.V. Zelentsov from the Moscow Physical Technical Institute for providing material for this study.

\section{References}

[1] P. Gütlich, A.B. Gaspar, V. Ksenofontov, Y. Garcia, J. Phys., Condens. Matter. 16, S1087 (2004).

[2] V. Ksenofontov, H. Spiering, A. Schreiner, G. Levchenko, H.A. Goodwin, P. Gütlich, J. Phys. Chem. Solids. 60, 393 (1999).

[3] M. Itoi, A. Taira, M. Enomoto, N. Matsushita, N. Kojima, Y. Kabayashi Asai, K. Koyama, T. Nakano, Y. Uwatoko, J. Yamaura, Solid State Commun. 130, 415 (2004).

[4] T. Tayagaki, K. Tanaka, H. Okamura, Phys. Rev. B 69, 064104 (2004).

[5] O. Kahn, C. J Martinez, Science 279, 44 (1998).

[6] V.A. Kogan, V.V. Zelentsov, G.M. Larin, V.V. Lukov, Transition Metal Complexes with Hydrazones. Physicochemical Properties and Structure, Nauka, Moscow 1990, p. 85 (in Russian).

[7] V.V. Zelentsov, G.M. Larin, E.V. Ivanov, N.V. Gerbeleu, A.V. Ablov, Teoret. Eksper. Chim. 7, 798 (1971) (in Russian).

[8] Yu.V. Yablokov, V.V. Zelentsov, M. AugustyniakJabłokow, A. Krupska, J. Mroziński, Mater. Sci. 21, 215 (2003).

[9] V.V. Zelentsov, Yu.V. Yablokov, M.A. AugustyniakJabłokow, A. Krupska, J. Mroziński, V.A. Ulanov, Chem. Phys. 301, 15 (2004).

[10] Yu.V. Yablokov, V.V. Zelentsov, M. AugustyniakJabłokow, A. Krupska, J. Mroziński, Magn. Res. Solids - electronic journal 6, 229 (2004).

[11] A. Krupska, M.A. Augustyniak-Jabłokow, V.Yu. Yablokov, V.V. Zelentsov, Acta Phys. Pol. A 108, 291 (2005).

[12] A. Krupska, M.A. Augustyniak-Jablokow, Yu.V. Yablokov, V.V. Zelentsov, V.A. Ulanov, J. Mrozinski, Acta Phys. Pol. A 110, 81 (2006).

[13] S. Hayami, K. Hiki, T. Kawahara, Y. Maeda, D. Urakami, K. Inoue, M. Ohama, S. Kawata, O. Sato, Chemistry 15, 3497 (2009).

[14] E.W.T. Yemeli, G.R. Blake, A.P. Douvalis, T. Bakas, G.O.R.A. van Ekenstein, P.J. van Koningsbruggen, Chem. Eur. J. 10, 1 (2010).

[15] A. Tissot, R. Bertoni, E. Collet, L. Toupet, M.-L. Boillot, J. Mater. Chem. 21, 18347 (2011).

[16] T. Buchen, D. Schollmeyer, P. Gütlich, Inorg. Chem. 35, 155 (1996).

[17] F. Varret, A. Bleuzen, K. Boukheddaden, A. Bousseksou, E. Codjovi, C. Enachescu, A. Goujon, J. Linares, N. Menendez, M. Verdaguer, Pure Appl. Chem. 74, 2159 (2002)

[18] T. Mitsuoka, M. Nakagawa, T. Iyoda, Y. Einaga, J. Nucl. Radiochem. Sci. 8, 1 (2007).

[19] M. Krupski, Rev. Sci. Instrum. 67, 2894 (1996).

[20] H. Liu, A. Fujishima, O. Sato, Appl. Phys. Lett. 85 , 2295 (2004).

[21] S. Floquet, E. Riviére, K. Boukheddaden, D. Morineau, M.-L. Boillot, Polyhedron, 2014 in press.

[22] A. Krupska, Acta Phys. Pol. A 124, 626 (2013). 